\title{
A Simplified Flexible Multibody Dynamics for a Main Landing Gear with Flexible Leaf Spring
}

\author{
Zhi-Peng Xue,, ${ }^{1,2}$ Ming Li, ${ }^{1}$ Yan-Hui Li, ${ }^{1}$ and Hong-Guang Jia ${ }^{1}$ \\ ${ }^{1}$ Changchun Institute of Optics, Fine Mechanics and Physics, Chinese Academy of Sciences, 3888, Dong Nanhu Road, \\ Changchun 430033, China \\ ${ }^{2}$ University of Chinese Academy of Sciences, 19, A Yuquan Road, Beijing 100049, China \\ Correspondence should be addressed to Hong-Guang Jia; 395227649@qq.com
}

Received 5 March 2014; Revised 1 July 2014; Accepted 6 July 2014; Published 23 July 2014

Academic Editor: Hassan Haddadpour

Copyright (C) 2014 Zhi-Peng Xue et al. This is an open access article distributed under the Creative Commons Attribution License, which permits unrestricted use, distribution, and reproduction in any medium, provided the original work is properly cited.

\begin{abstract}
The dynamics of multibody systems with deformable components has been a subject of interest in many different fields such as machine design and aerospace. Traditional rigid-flexible systems often take a lot of computer resources to get accurate results. Accuracy and efficiency of computation have been the focus of this research in satisfying the coupling of rigid body and flex body. The method is based on modal analysis and linear theory of elastodynamics: reduced modal datum was used to describe the elastic deformation which was a linear approximate of the flexible part. Then rigid-flexible multibody system was built and the highly nonlinearity of the mass matrix caused by the limited rotation of the deformation part was approximated using the linear theory of elastodynamics. The above methods were used to establish the drop system of the leaf spring type landing gear of a small UAV. Comparisons of the drop test and simulation were applied. Results show that the errors caused by the linear approximation are acceptable, and the simulation process is fast and stable.
\end{abstract}

\section{Introduction}

Automatic takeoff, landing, and taxiing are very important parts of completely autonomous flight of UAV, and taxiing tests on UAVs are obviously expensive and risky as it is easy to bring about accidents under high-speed taxiing. Simulation on the other hand is particularly important before the prototype taxiing experiment. During the development of control systems, real-time simulations such as man-in-theloop (MIL) and hardware-in-the-loop (HIL) are also used to take place between design level simulations and costly experiments with the real plant [1-3]. Obviously accurate and efficient computer simulations of UAV on-ground models play an important role in the control system design, performance evaluations, and dynamics analysis of such vehicle systems. Meanwhile research on ground dynamics in the past decades has achieved significant results, and the design of these dynamics traditionally relies on mathematical model.
Ro [4] has studied aircraft-runway dynamics in detail. In general mathematical equations of ground dynamics are complex, highly coupled, and nonlinear; their derivation and numerical implementation demand considerable time and computer cost. In recent years, the accuracy and computational efficiency of flexible multibody has been the focus of research $[5,6]$. The simplified flexible multibody method enables the dynamic response to simulate effectively, even be used in many real-time simulations [7-9].

Nonretractable type landing gear has been widely used in small UAVs, a leaf spring is used to provide stiffness and damping which absorbs shock and energy by its space deformations $[10,11]$. A common way to model body flexibility is the finite element (FE) method which is based on body geometry and can solve deformations stress and strain precisely $[12,13]$. But the finite element method often produces a large number of degrees of freedom (DOF) which leads to the computer computation very costly [14]. To reduce 
the amount of DOF a simplified method was applied which is called modal method that a body's deformation can be approximated by a linear combination of preselected mode shape functions [15]. Hauser et al. [7] have studied the computational efficiency and error of modal method, and it has been applied in video games, surgery training, and other simulation systems. In this paper modal superposition method was used to establish the flexible model of the leaf spring landing gear.

Flexible body, experiencing large space motion while undergoing small elastic deformations, theoretically will cause the inertial coupling between elastic deformation and rigid body motion; the most common approach is to use a time-varying mass matrix which is highly nonlinear and needs to be recomputed in every time step. Many methods [16] are used to simplify the mass matrix. A more simple way is called the linear theory of elastodynamics that the impact of elastic deformation at the multibody dynamics is ignored and may not be suitable for some cases like high-speed rotation.

Tire is another elastic part of the system. The most representative models are based on empirical functions, designed and tuned to fit measure data. Some mathematical formulations can be already found in recent research. Pacejka’s Magic Formula (trigonometric formulation) [17] is a popular model in the automotive industry. The NASA, through an AGARD project, proposed a mathematical formulation applied to commercial airplanes and based on polynomial equations $[3,18]$. Linear parameter varying (LPV) and linear fractional transformation (LFT) modeling are also used together with a novel force identification approach based on nonlinear dynamic inversion theory to obtain a simplified tire model [19]. To decrease the complexity of the model a typical, widely used tire model is a point contact spring model with either linear or nonlinear stiffness and damping in parallel [4].

The idea which motivates this work is to propose a general modeling method for elastic leaf spring. This paper also proposes a simplified flexible multibody to study the dynamics of a leaf spring landing gear, aiming at efficient formulation and solution of equations where a compromise between accuracy and computational efficiency has to be reached. Another contribution of this paper is to discuss the computer implementation of the proposed formulation and demonstrate its use by developing a drop system model. Drop test and simulation are conducted to analyze the model accuracy and efficiency.

\section{Flexible Model}

Modal method is a linear approximation of elastic deformation; complex nonlinear differential equations are decoupled into a set of single DOF linear equations by modal coordinate transformation. These equations can be further transformed into a more briefly state space form as shown in Figure 1. Since the decoupled equations can be solved by computer directly, the main advantage of modal method is that its computational efficiency is very high.

2.1. Modal Superposition. Continuum body can be described by its mass matrices $M_{u}$ and stiffness matrices $K_{u}$, with a

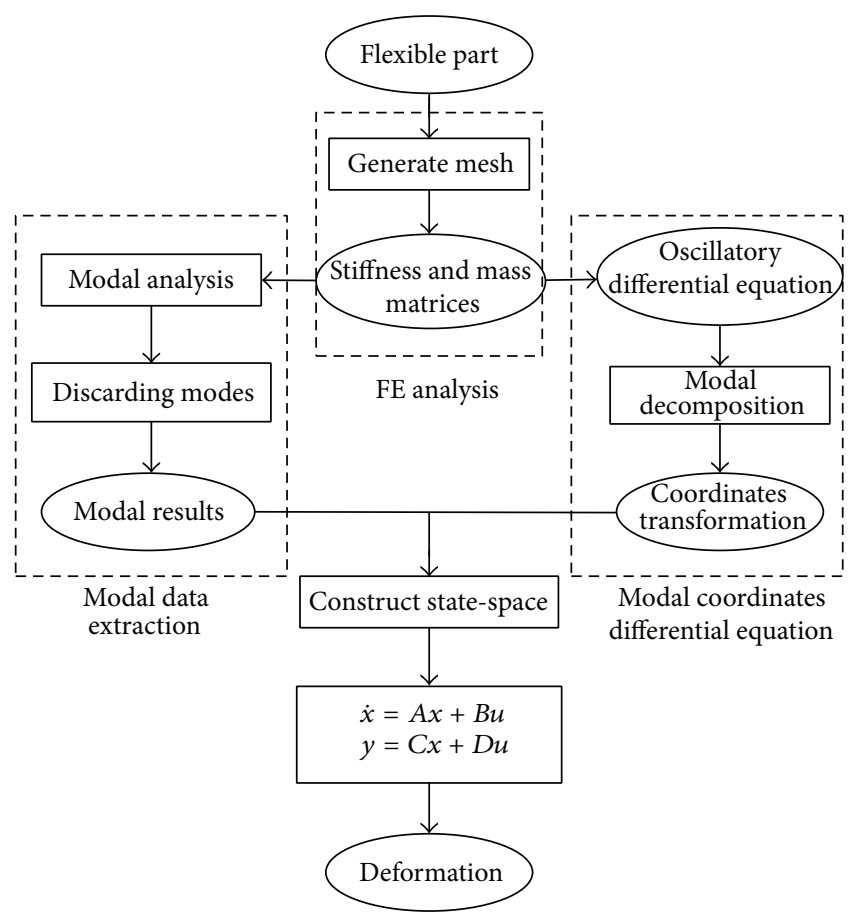

FIGURE 1: Block diagram of modal analysis method.

damping matrix $C_{u}$; then the general vibration differential equation is as

$$
M_{u} \ddot{u}_{f}+C_{u} \dot{u}_{f}+K_{u} u_{f}=f,
$$

where $u_{f}$ represents the displacements vector of the node degrees of freedom (DOF), $f$ represents the force vector, and generally $u_{f}$ has a large amount of DOF $n$. The modal coordinate transformation as shown in (2) is used to decouple vibration differential equations into linear equations:

$$
u_{f}=\Phi q_{f},
$$

where $q_{f}$ represents the modal coordinates and $\Phi$ represents the reduced modal matrix. By substituting (2) into (1) and premultiplying the transpose of matrix $\Phi$, one gets the following reduced system with the dimension $n_{m}\left(n_{m}<n\right)$ :

$$
M_{q} \ddot{q}_{f}+C_{q} \dot{q}_{f}+K_{q} q_{f}=Q
$$

in which

$$
\begin{gathered}
M_{q}=\Phi^{T} M \Phi=I_{n_{m} \times n_{m}}, \\
K_{q}=\Phi^{T} K \Phi=\operatorname{diag}\left(\omega_{i}^{2}\right), \\
C_{q}=\Phi^{T} C \Phi, \\
Q=\Phi^{T} f,
\end{gathered}
$$

where $\omega_{i}$ is the natural frequency of the $i$ th mode $(1 \leq$ $i$ th $\left.\leq n_{m}\right), M_{q}$ is identity matrix, and $K_{q}$ and $C_{q}$ are diagonal matrices. Thus, the vibration differential equations are approximated by (3). Each modal coordinate can be calculated independently; then modal coordinates (Figure 2) are used to solve for node displacements using (2). 


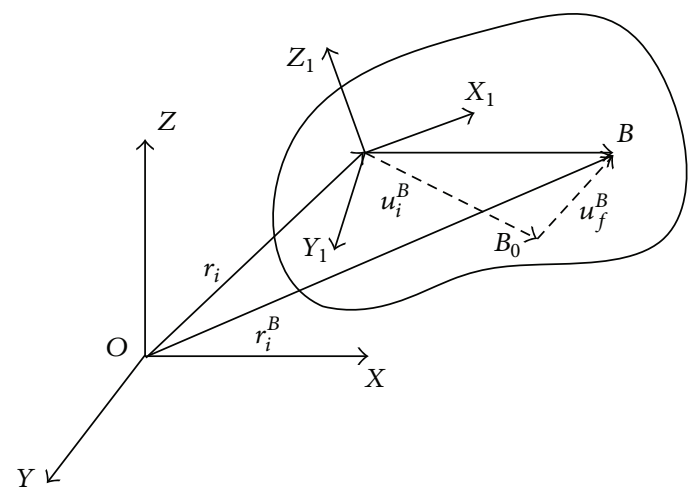

FIgURE 2: Coordinates of flexible body.

2.2. State-Space Form. The mathematical calculations through matrix-vector form by computer will be very efficient by transforming (3) into the state space form. The linear state space is as follows:

$$
\begin{aligned}
& \dot{x}=A x+B u, \\
& y=C x+D u,
\end{aligned}
$$

where $x$ is the state vector as (6) and $\dot{x}$ is the derivative of the state vector. $A, B, C$, and $D$, respectively, are the system matrix, input matrix, output matrix, and transfer matrix; $u$ and $y$ are the $\mathrm{I} / \mathrm{O}$ of system:

$$
x=\left[\begin{array}{c}
q_{f} \\
\dot{q}_{f}
\end{array}\right] .
$$

Then (3) is transformed as follows:

$$
\begin{gathered}
\ddot{q}_{f}+2 \Gamma \dot{q}_{f}+\Omega q_{f}=\Sigma u, \\
\Sigma=\Phi^{T} F
\end{gathered}
$$

where $\Sigma$ is defined in (8); $F$ is used to define the degrees of freedom of the system input.

According to (5), the displacements, velocities, and accelerations are described by the modal coordinates:

$$
y=\left[\begin{array}{c}
\Theta q_{f} \\
\Theta \dot{q}_{f} \\
\Theta \ddot{q}_{f}
\end{array}\right], \quad \Theta=T \Phi,
$$

where matrix $T$ is used to define the system output. Then each matrix of state space can be determined according (7):

$$
\begin{array}{ccc}
A=\left[\begin{array}{cc}
0 & I \\
-\Omega & -2 \Gamma
\end{array}\right], & B=\left[\begin{array}{l}
0 \\
\Sigma
\end{array}\right], \\
C=\left[\begin{array}{cc}
\Theta & 0 \\
0 & \Theta \\
-\Theta \Omega & -2 \Theta \Gamma
\end{array}\right], & D=\left[\begin{array}{c}
0 \\
0 \\
\Theta \Sigma
\end{array}\right] .
\end{array}
$$

The matrices of state space are constructed by the reduced modal data, its output is the deformation, and input is the stress of the flexible body.
2.3. Modal Reduction. One gets the same number of modes with the number of DOF by modal analysis, and the accuracy of modal method depends on a user-defined frequency range, though the more modal modes are contained, the greater cost of calculation is required. Modal reduction is based on the frequency response analysis which is used to determine the contribution of each mode to the displacement response.

Taking Laplace transforms with zero initial conditions of (5),

$$
\begin{gathered}
x(s)=(s I-A)^{-1} B u(s), \\
y(s)=C(s I-A)^{-1} B u(s)+D u(s) .
\end{gathered}
$$

Solving the transfer functions

$$
\frac{y(s)}{u(s)}=C(s I-A)^{-1} B+D
$$

for combinations of degrees of freedom where forces are applied and where displacements are taken can get the amplitude of the response of each mode.

\section{Rigid-Flexible Multibody Dynamics}

3.1. Multibody Dynamics. The most frequent approach used in multibody system to consider flexibility is the floating frame of reference formulation (FFRF), in which two sets of coordinate are used to describe a deformable body as discussed by Shabana [20]. One set is reference coordinate which describes the location and orientation of a selected body coordinate system, while the second one is elastic coordinate which describes the deformation of the body with respect to its coordinates. The global position of an arbitrary point $B$ on the flexible body is as follows:

$$
r_{i}^{B}=r_{i}+A_{i}\left(u_{i}^{B}+u_{f}^{B}\right)
$$

where $r_{i}$ is the local position vector, $A_{i}$ is the transformation matrix, $u_{i}^{B}$ is the local position of point $B$ in the undeformed state, and $u_{f}^{B}$ is the deformation vector of point $B$ which can be approximated in the modal coordinate using (2).

Thus, we can define the coordinates of body $i$ as

$$
q^{i}=\left[\begin{array}{c}
R^{i} \\
\theta^{i} \\
q_{f}^{i}
\end{array}\right],
$$

where $R^{i}$ and $\theta^{i}$ are the reference coordinates respect displacement and rotation, respectively; $q_{f}^{i}$ is the vector of elastic coordinates. 
The equations of motion of Lagrangian formalism in multibody systems have been proposed in [20]. The augmented matrix equation expressed in generalized coordinates is

$$
\begin{gathered}
{\left[\begin{array}{ccc}
m_{R R}^{i} & m_{R \theta}^{i} & m_{R f}^{i} \\
& m_{\theta \theta}^{i} & m_{\theta f}^{i} \\
\text { symmetric } & & m_{f f}^{i}
\end{array}\right]\left[\begin{array}{c}
\ddot{R}^{i} \\
\ddot{\theta}^{i} \\
\ddot{q}_{f}^{i}
\end{array}\right]+\left[\begin{array}{ccc}
0 & 0 & 0 \\
0 & 0 & 0 \\
0 & 0 & K_{f f}^{i}
\end{array}\right]\left[\begin{array}{c}
R^{i} \\
\theta^{i} \\
q_{f}^{i}
\end{array}\right]} \\
+\left[\begin{array}{c}
C_{R^{i}}^{T} \\
C_{\theta^{i}}^{T} \\
C_{q_{f}^{i}}^{T}
\end{array}\right] \lambda=\left[\begin{array}{l}
\left(Q_{e}^{i}\right)_{R} \\
\left(Q_{e}^{i}\right)_{\theta} \\
\left(Q_{e}^{i}\right)_{f}
\end{array}\right]+\left[\begin{array}{l}
\left(Q_{v}^{i}\right)_{R} \\
\left(Q_{v}^{i}\right)_{\theta} \\
\left(Q_{v}^{i}\right)_{f}
\end{array}\right]
\end{gathered}
$$

under the constraints

$$
C\left(R, \theta, q_{f}\right)=0
$$

where $m_{i i}$ represents mass submatrices, $K_{f f}$ is the stiffness matrix, $\lambda$ is the vector of Lagrange multiplier, matrix $C_{q}^{T}$ represents the transposition of the constraint Jacobian matrix, $Q_{e}$ is the vector of external force, and $Q_{v}$ is the vector of quadratic velocity.

\subsection{Coupling between Reference and Elastic Displacements}

3.2.1. Inertia of Deformable Bodies. The following definition of the kinetic energy is used:

$$
T^{i}=\frac{1}{2} \int_{V^{i}} \rho^{i} \dot{r}_{i}^{B^{T}} \dot{r}_{i}^{B} d V^{i}
$$

where $T^{i}$ is the kinetic energy of body $i ; \rho^{i}$ and $V^{i}$ are, respectively, the mass density and volume of body $i$; and $\dot{r}_{i}^{B}$ is the global velocity vector of point $B$ on the body. The expression of $\dot{r}_{i}^{B}$ is

$$
\dot{r}_{i}^{B}=\dot{r}_{i}+\dot{A}_{i}\left(u_{i}^{B}+u_{f}^{B}\right)+A_{i}\left(\dot{u}_{i}^{B}+\dot{u}_{f}^{B}\right),
$$

where $\dot{u}_{i}^{B}=0$ is used, the central term on the right-hand side of (18) can in general be written as

$$
\dot{A}_{i}\left(u_{i}^{B}+u_{f}^{B}\right)=B_{i} \dot{\theta}_{i}
$$

where $\dot{\theta}_{i}$ is the vector of the time derivatives of the rotational coordinates of the body reference and $B^{i}$ is defined as

$$
B_{i}=\left[\frac{\partial}{\partial \theta_{1}^{i}} A_{i}\left(u_{i}^{B}+u_{f}^{B}\right) \cdots \frac{\partial}{\partial \theta_{n_{r}}^{i}} A_{i}\left(u_{i}^{B}+u_{f}^{B}\right)\right],
$$

where $n_{r}$ is the total number of rotation coordinates of the reference of body $i$. So (18) can be written as

$$
\dot{r}_{i}^{B}=\left[\begin{array}{lll}
I & B_{i} & A_{i} \Phi_{i}
\end{array}\right]\left[\begin{array}{c}
\dot{r}_{i} \\
\dot{\theta}_{i} \\
\dot{q}_{f}^{i}
\end{array}\right] .
$$

Equation (17) can be written in a more compact form as

$$
T^{i}=\frac{1}{2} \dot{q}_{i}^{T} M^{i} \dot{q}^{i}
$$

The expression of mass matrix of body $i$ is

$$
\begin{aligned}
M_{i} & =\int_{V^{i}} \rho^{i}\left[\begin{array}{c}
I \\
B_{i}^{T} \\
\left(A_{i} \Phi_{i}\right)^{T}
\end{array}\right]\left[\begin{array}{lll}
I & B_{i} & A_{i} \Phi_{i}
\end{array}\right] d V^{i} \\
& =\int_{V^{i}} \rho^{i}\left[\begin{array}{ccc}
I & B_{i} & A_{i} \Phi_{i} \\
& B_{i}^{T} B_{i} & B_{i}^{T} A_{i} \Phi_{i} \\
\text { symmetric } & & \Phi_{i}^{T} \Phi_{i}
\end{array}\right] d V^{i} .
\end{aligned}
$$

The mass matrix can also be written as

$$
M_{i}=\left[\begin{array}{ccc}
m_{R R}^{i} & m_{R \theta}^{i} & m_{R f}^{i} \\
& m_{\theta \theta}^{i} & m_{\theta f}^{i} \\
\text { symmetric } & & m_{f f}^{i}
\end{array}\right] .
$$

As given by (23) and (24), the submatrix $m_{R R}^{i}$ associated with the translations of the origin of the body reference is a constant matrix; the submatrices $m_{R f}^{i}$ and $m_{\theta f}^{i}$ represent the coupling between reference motion and elastic deformation; and submatrices $m_{\theta \theta}^{i}$ and $m_{R \theta}^{i}$ depend on both the rotational reference coordinates and the elastic coordinates of the body i. As (20) shows

$$
B_{i}=B_{i}\left(\dot{\theta}_{i}, \dot{q}_{f}^{i}\right)
$$

If the body is rigid and the origin of the body reference is attached to the center of mass, the translation and rotation of the rigid body are dynamically decoupled. In this case the matrix $m_{R \theta}^{i}$ is the null matrix which means that the matrix $B_{i}$ is also the null matrix. This, however, is not the case when deformable bodies are considered. As (25) shows, there is no guarantee that $B_{i}$ is the null matrix because of the time-dependent coordinates $\dot{q}_{f}^{i}$. Then vector $B_{i}$ must be iteratively updated. Therefore, submatrices $m_{R \theta}^{i}, m_{\theta f}^{i}, m_{R f}^{i}$, and $m_{\theta \theta}^{i}$ must be iteratively updated, respectively. Finally, the submatrix $m_{f f}^{i}$ in (24) is independent of the generalized coordinates of the body, therefore, is constant.

3.2.2. Linear Theory of Elastodynamics. As above section shows, the mixed set of reference and elastic coordinates of deformable body leads to a highly nonlinear mass matrix as a result of the inertia coupling between the reference and the elastic displacements. The mass matrix must be iteratively updated.

Rigid body motion and flexible deformation interact with each other though inertial effects. The deformation body is rotating rapidly; neither effect will be significant. Therefore a solution strategy that has been used to omit these effects. The multibody system was first as rigid body systems as (26). General multi-rigid-body computer programs then be used to solve for the inertia and reaction forces. These forces are then introduced to the state space form of elastic deformable 


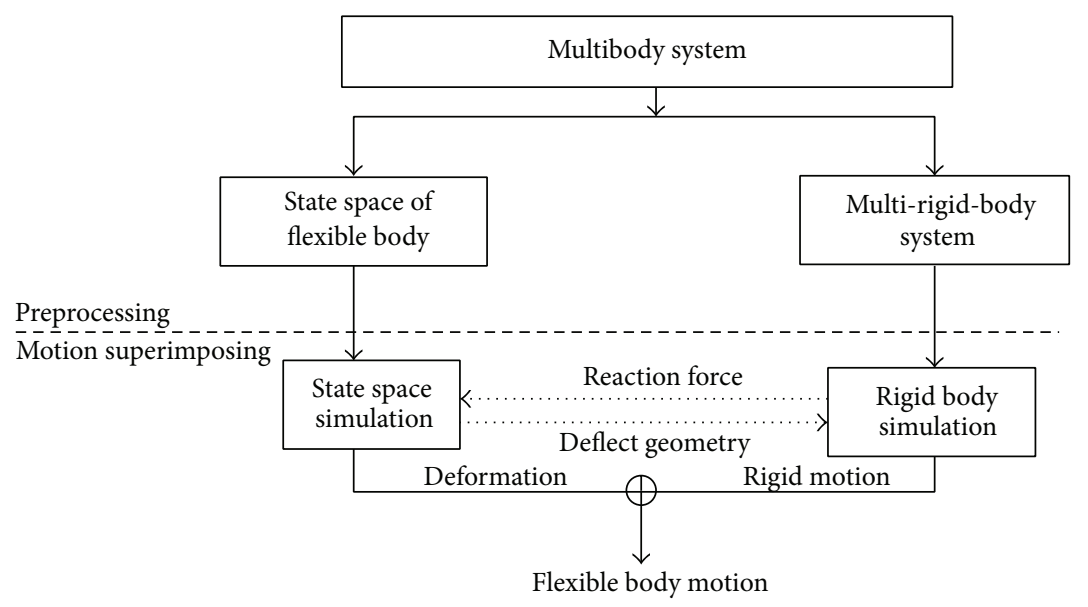

FIGURE 3: Block diagram of rigid-flexible multibody system.

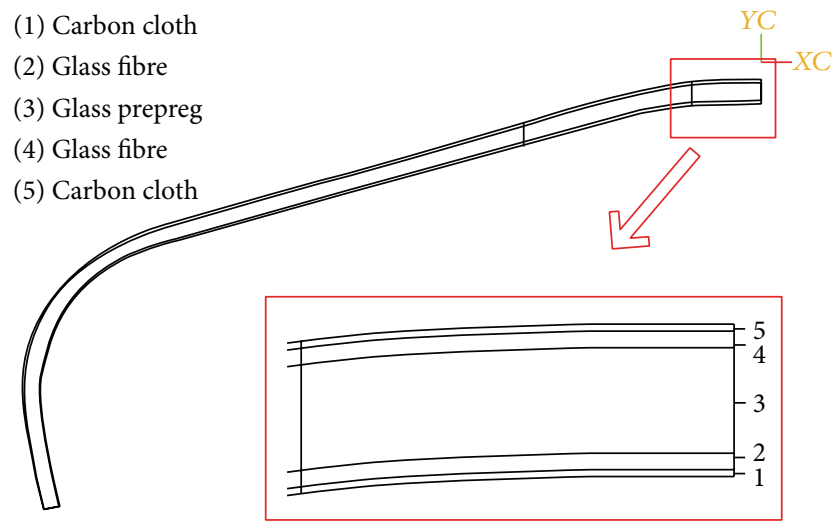

FIGURE 4: Five-layer leaf spring landing gear.

model to solve the deformation displacements. The total motion of a body is then obtained by superimposing the small elastic deformation on the gross rigid body motion as shown in Figure 3:

$$
\left[\begin{array}{ll}
m_{R R}^{i} & \\
& m_{\theta \theta}^{i}
\end{array}\right]\left[\begin{array}{c}
\ddot{R}^{i} \\
\ddot{\theta}^{i}
\end{array}\right]+\left[\begin{array}{c}
C_{R^{i}}^{T} \\
C_{\theta^{i}}^{T}
\end{array}\right] \lambda=\left[\begin{array}{c}
\left(Q_{e}^{i}\right)_{R} \\
\left(Q_{e}^{i}\right)_{\theta}
\end{array}\right]+\left[\begin{array}{c}
\left(Q_{v}^{i}\right)_{R} \\
\left(Q_{v}^{i}\right)_{\theta}
\end{array}\right] .
$$

\section{Simulation and Test}

Sections above have presented a very effective modeling method for rigid-flexible multibody system which will be used in this section to build the main landing gear of a small UAV in Matlab/Simulink. Tests are implemented to verify the accuracy of this method.

4.1. Modeling of the Leaf Spring. Cantilever type leaf spring was manufactured by composite material layers as shown in Figure 4 and the parameters of each material are shown in

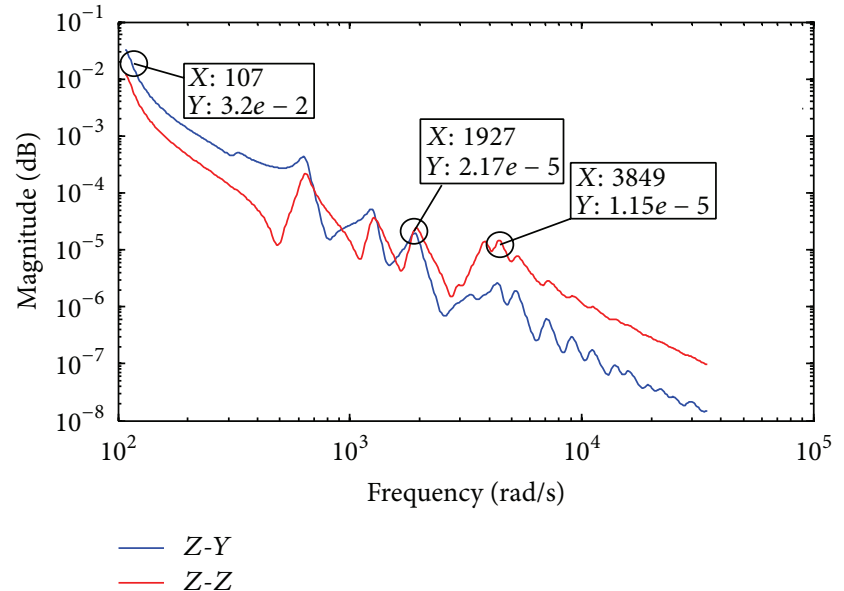

FIGURE 5: Frequency response.

TABLE 1: Layer parameters.

\begin{tabular}{lccc}
\hline Parameters & \multicolumn{3}{c}{ Materials } \\
& Carbon cloth & Glass fibre & Glass prepreg \\
\hline Thickness $(\mathrm{mm})$ & 0.2 & 0.2 & 0.2 \\
Young's modulus $E_{x}(\mathrm{Gpa})$ & $57-66$ & $17-20$ & $42-52$ \\
Young's modulus $E_{y}(\mathrm{Gpa})$ & $57-66$ & $17-20$ & $8-10$ \\
Young's modulus $E_{z}(\mathrm{Gpa})$ & $8-10$ & $8-10$ & $8-10$ \\
Shear modulus $G(\mathrm{Gpa})$ & 4.8 & 4 & 3.5 \\
Poisson's parameters & 0.3 & 0.3 & 0.3 \\
Destiny $\left(\mathrm{g} / \mathrm{cm}^{3}\right)$ & 1.6 & 2 & 2 \\
\hline
\end{tabular}

Table 1. The FE model of leaf spring has 2868 nodes which means that it has $2868 \times 3$ modes. The first 6 modes are rigid body modes resulting from a free-free modal analysis and the 7 th to 17 th modes are shown in Table 2 .

Frequency response analysis for first 50 modes except the rigid body modes is performed at the joint connected the landing gear and tire. The results are shown in Figure 5, where $Z-Z$ means the response of vertical when vertical input 

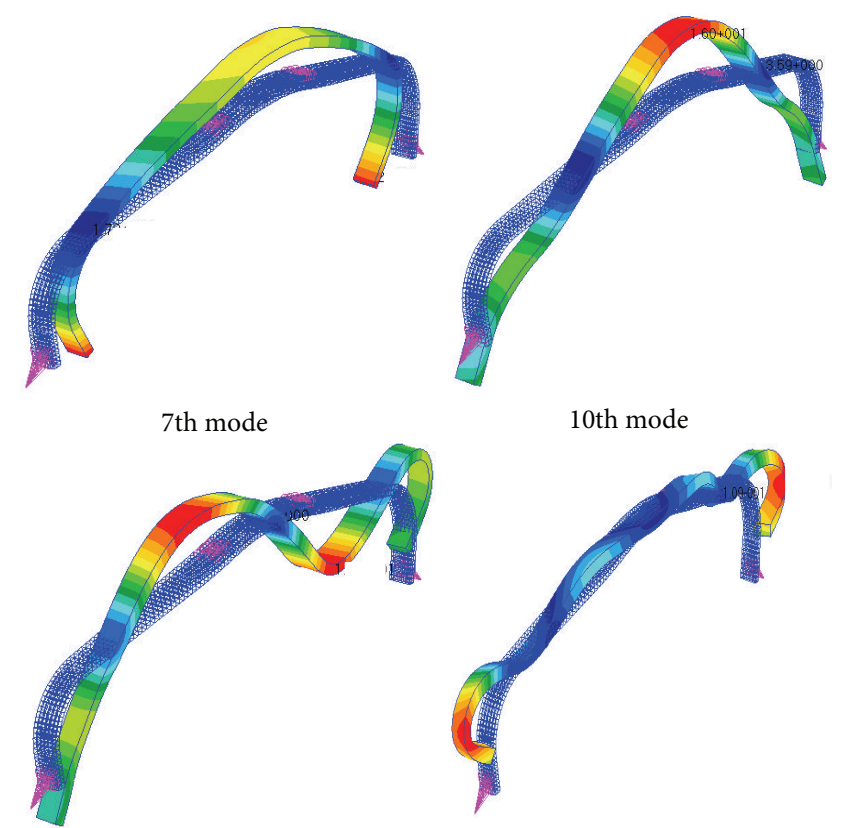

11th mode

17th mode

Figure 6: Parts of mode shapes.

and $Z-Y$ means the response of lateral when vertical input. The magnitude of lateral response ( $Y$ direction) is greater than that of vertical response ( $Z$ direction) at the former modes, which means that the lateral stiffness is lower than the vertical stiffness. For the reduced modes must include the major modes of the two directions, mode reduce is based on $Z-Z$.

The 7th mode has the largest frequency magnitude, and, as shown in Figure 6, 7th mode shape is the first bending mode; this bending shape was appearing repeatedly during the compressing of the leaf spring of drop tests, which indicated that the 7th mode is the most important mode. The magnitude response decreases as the frequency increases, and the first area peak appears at the 10th mode, and its mode shape is second bending mode. The mode which has a resonant area peak is the important mode, such as the 11th mode; it is the third bending mode. The magnitude of the 17th mode is about $10^{3}$ times smaller than that of the 7 th mode, and the magnitude of the resonant peaks after 17th modes is far smaller than that of the 17 th mode. So the 7 th to 17 th modes are the major modes.

Then 11 modes are used to construct the elastic model, and each mode shape of the reduced modes still contains the modal datum of every node. For we only concern the nodes where forces are applied and where displacements are taken, the nodes except these located on the joints and where we want to measure the deformations are excluded. So the flexible model is constructed by the reduced modes and reduced nodes.

4.2. Rigid-Flexible Multibody Model. A penetration formulation is used to calculate the normal force between the tire and
TABLE 2: Modal analysis results.

\begin{tabular}{lc}
\hline Modes & Frequency rad/s \\
\hline 7 & 107 \\
8 & 322 \\
9 & 494 \\
10 & 641 \\
11 & 1261 \\
12 & 1294 \\
13 & 1927 \\
14 & 2945 \\
15 & 3393 \\
16 & 3453 \\
17 & 3849 \\
\hline
\end{tabular}

ground as shown in Figure 7(a). Similar to a spring/damper formula, the force applied to the tire is proportional to the penetration depth and the penetration rate. The normal force is described as

$$
F_{\text {nom }}=K_{\text {nom }} \tau+C_{\text {nom }} \dot{\tau},
$$

where $K_{\text {nom }}$ and $C_{\text {nom }}$ are, respectively, the vertical stiffness and damping of tire. $\tau$ is the penetration depth.

The lateral force exerted by the tires is described as a function of slip angle. As shown in Figure 7(b), slip angle $\beta$ is the orientations of the velocity of the tire's points of contact the surface of motion with respect to the tires' longitudinal axes. The lateral force is applied to account for the side loading on the tire. It is applied in a direction aligned with the rotation axis of the wheel. This force acts in the opposite direction to the lateral motion of the wheel and can be written as

$$
F_{\text {lat }}=C_{1} \beta \text {, }
$$

where $C_{1}$ is the lateral stiffness factors and $\beta$ is the slip angle. The measured data of tire parameters are shown in Table 3 .

The drop multibody system includes leaf spring, load masses, and tires as in Figure 8. The leaf spring and load masses are connected by 2 pin joints a and $b$, the leaf spring and tires are connected by 2 revolute joints $\mathrm{c}$ and $\mathrm{d}$, and the landing gear system is connected with ground by a prismatic joint.

As introduced in Section 3.2, both rigid model and flexible model are used to build the leaf spring, and the information exchanged between them is reaction forces and deformations; the external forces of the flexible model are the reaction forces of the joints. Massless bodies are used to transfer information between rigid body and flexible body. The entire model experiences free falling if the tires have not contacted the ground. If contact happens, reaction forces are solved by rigid system simulation; then these forces are as the inputs of the state space to calculate the displacements of the joint nodes which are used to correct the global positions of these joint nodes in the next simulation step; rigid-flexible multibody simulation is accomplished as this iterative loop. 


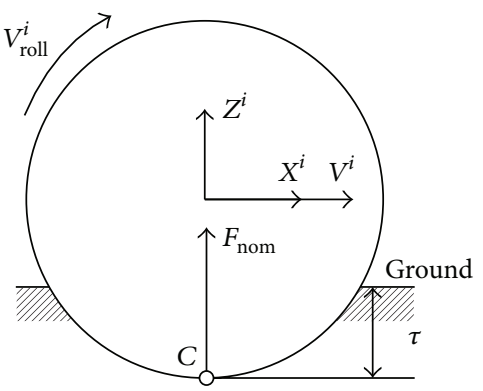

(a) Vertical force

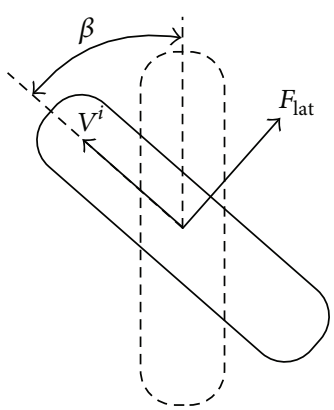

(b) Lateral force

Figure 7: Tire model.

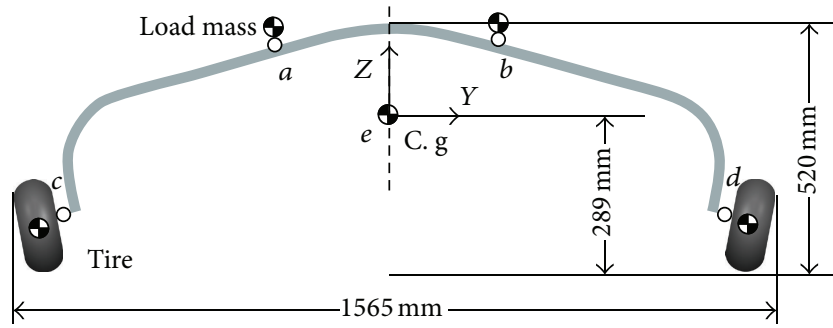

FIGURE 8: Drop multibody system.

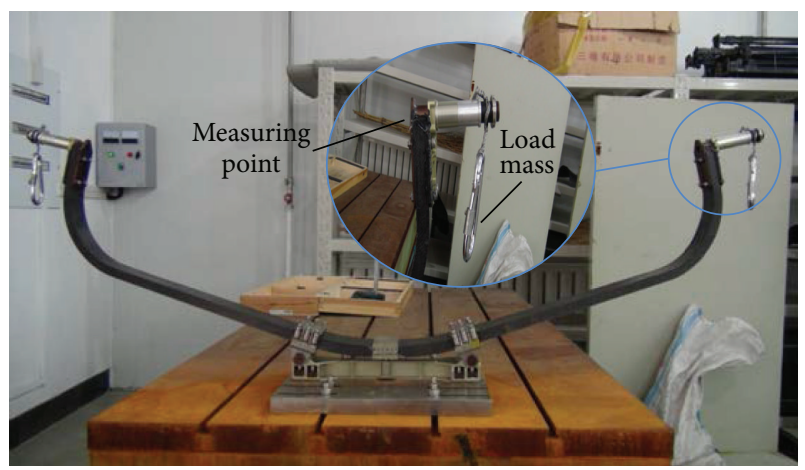

Figure 9: Statics test.

4.3. Statics Test. Statics test was conducted before the drop test as shown in Figure 9. As the results show in Table 4, the errors of vertical displacement are below 3\% for the statics conditions. So the present flexible model of leaf spring leads to excellent accuracy under the statics situation.

4.4. Drop Test. As shown in Figure 10(a), the drop test system includes supporting subsystem, slide track subsystem, lift subsystem, and data acquisition subsystem. The drop mass $(100 \mathrm{~kg})$ is attached to the top of the landing gear to simulate the landing mass. For the test results are used to compare with the simulation results, so only the vertical velocity is considered.
TABLE 3: Tire parameters.

\begin{tabular}{lc}
\hline Parameter & Value \\
\hline Radius & $0.1 \mathrm{~m}$ \\
Width & $0.075 \mathrm{~m}$ \\
Vertical stiffness & $75000 \mathrm{~N} / \mathrm{m}$ \\
Lateral stiffness & $166.55 \mathrm{~N}{ }^{\circ}$ \\
Mass & $1.5 \mathrm{~kg}$ \\
$I_{x x} / I_{y y}$ & $3.45 * 10^{-3} \mathrm{Kgm}^{2}$ \\
$I_{z z}$ & $5.62 * 10^{-3} \mathrm{Kgm}^{2}$ \\
Damping & $175 \mathrm{~N} /(\mathrm{m} / \mathrm{s})$ \\
\hline
\end{tabular}

TABLE 4: Statics results.

\begin{tabular}{lccc}
\hline Load mass & Tests $(\mathrm{mm})$ & Simulations $(\mathrm{mm})$ & Errors \\
\hline $10 \mathrm{~kg}$ & 5.125 & 5.079 & $-0.9 \%$ \\
$20 \mathrm{~kg}$ & 10.250 & 10.11 & $-0.6 \%$ \\
$30 \mathrm{~kg}$ & 15.750 & 15.754 & 0 \\
$40 \mathrm{~kg}$ & 21.000 & 21.4145 & $2 \%$ \\
$50 \mathrm{~kg}$ & 26.625 & 27.3015 & $2.5 \%$ \\
\hline
\end{tabular}

TABle 5: Parameters of drop tests.

\begin{tabular}{lcccc}
\hline Number Drop velocity & Load mass & Drop height & Drop attitude \\
\hline 1 & $1.8 \mathrm{~m} / \mathrm{s}$ & $180 \mathrm{~kg}$ & $0.165 \mathrm{~m}$ & Level \\
2 & $3 \mathrm{~m} / \mathrm{s}$ & $180 \mathrm{~kg}$ & $0.46 \mathrm{~m}$ & Level \\
\hline
\end{tabular}

According to the use-velocity and limit-velocity of landing, set the drop heights as Table 5; the tires are still at the beginning of the drop test.

Video footage is used to capture the vertical and lateral displacements during drop tests by the vertical and lateral fixed rulers.

4.5. Results. The flexible body motion of landing gear is shown in Figure 11. Results including the displacements and 


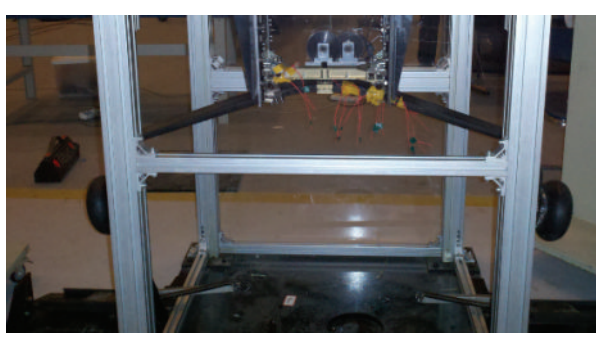

(a) Drop test system

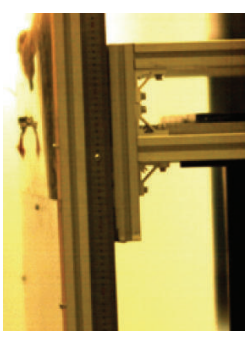

(b) Measuring system

Figure 10: Devices of drop test system.

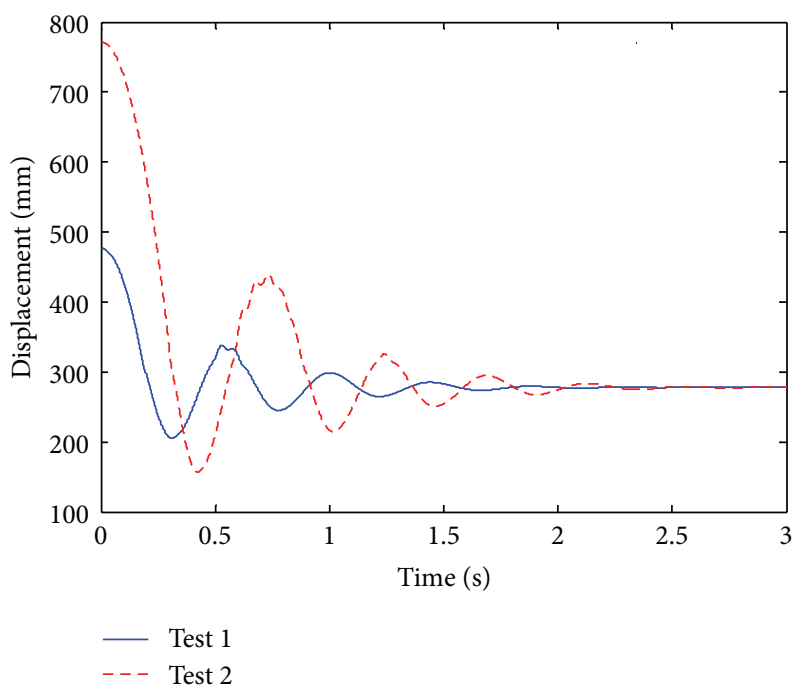

FIGURE 11: Motions of joint $\mathrm{b}$ of landing gear.

TABLE 6: Displacements of joint $b$ in test $2(\mathrm{~mm})$.

\begin{tabular}{lcc}
\hline Drop test & Simulation & Error \\
\hline-155.4 & -141.9 & $8.7 \%$ \\
122.5 & 132.7 & $8.3 \%$ \\
-61.3 & -64.7 & $5.5 \%$ \\
26.6 & 28.7 & $7.9 \%$ \\
-10.8 & -11.4 & $5.6 \%$ \\
\hline
\end{tabular}

reaction forces of simulation 2 are shown in Figure 12. The simulation step is $1 \mathrm{~ms}$. It is because of the loss of energy during impact which is caused by the frictions of drop test that the shock time of drop test is shorter than that of the drop simulation.

In test 2 the vertical displacements of joint $b$ from the measuring results of video footage and simulation results when the landing gear undergoing fully compressed are given in Table 6 . In which a negative displacement means the landing gear is under fully compress and a positive displacement means the landing gear is on the rebound.

As shown in Table 6 the landing gear absorbed majority energy after 3 compressions, and the errors are less than $10 \%$.
TABLE 7: Error of elastic deformations of joint $\mathrm{d}$ in test $2(\mathrm{~mm})$.

\begin{tabular}{lcc}
\hline Compression number & Vertical & Lateral \\
\hline 1 & $8.0 \%$ & $7.6 \%$ \\
2 & $6.4 \%$ & $6.1 \%$ \\
3 & $5.3 \%$ & $4.9 \%$ \\
\hline
\end{tabular}

Elastic deformations of joint $\mathrm{d}$ during the three compressions are shown in Table 7.

\section{Conclusions}

A simplified rigid-flexible multibody method is presented and used in modeling the main landing gear system of a small UAV. The computational efficiency of the vibration differential equations which has been decoupled by modal coordinate transformation is very high. Modal superposition has greatly reduced the model that only 11 modes are used to build the flexible model of the leaf spring, while the FE model has 17208 DOFs. The highly nonlinear mass matrix because of the finite rotation of the flexible part is eliminated by linear elastic dynamics. So the whole model is characterized by a very small number of equations, and there are no significant nonlinear factors that a very high computational efficiency can be reached and it is very suitable for real-time simulation systems.

The comparisons between test and simulations are applied to verify the accuracy of this approach. Static errors are less than $5 \%$, and dynamics errors are less than $10 \%$; meanwhile the simulation step has reached to $1 \mathrm{~ms}$. So the computation of this approach is accuracy and efficiency. The modal datum used by this approach can be get from not only the FE modal analysis, but also the modal experiments to modify the first few modes, to get a more accurate and reliable result. Results have shown that the proposed method has a good potential to model UAV with elastic leaf spring landing gear on-ground multibody system which can be used in realtime simulations such as MIL and HIL simulations in the future.

\section{Conflict of Interests}

The authors declare that there is no conflict of interests regarding the publication of this paper. 


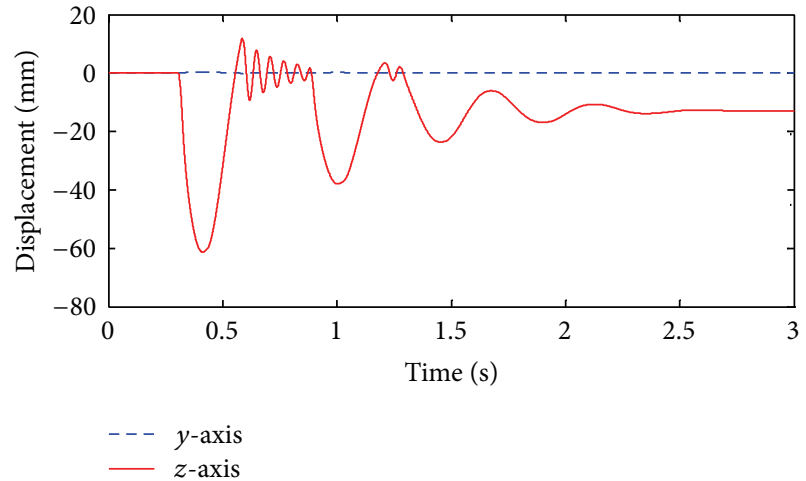

(a) Displacement of joint b

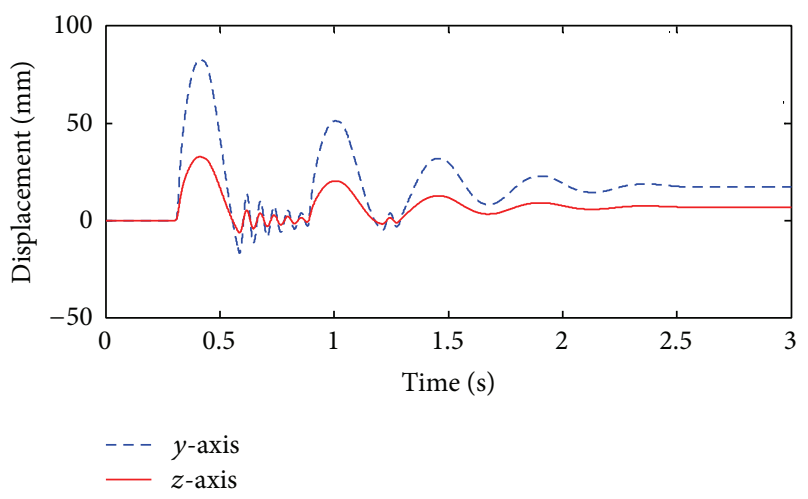

(c) Displacement of joint d

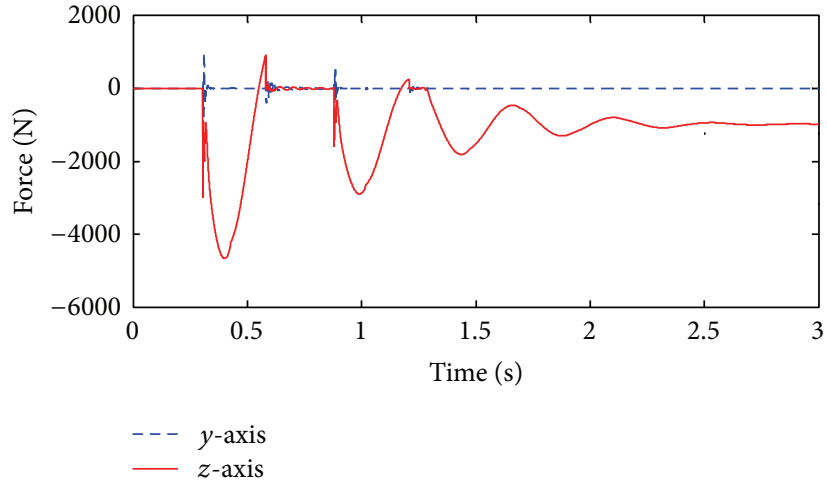

(b) Reaction force of joint $b$

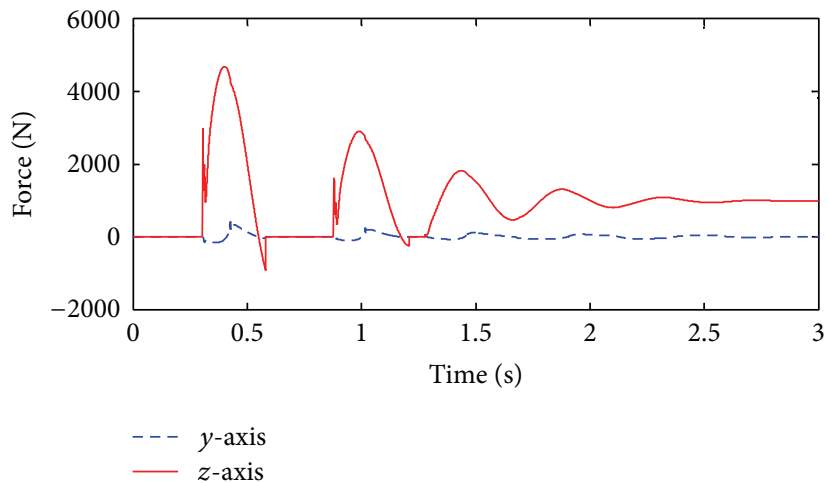

(d) Reaction force of joint $\mathrm{d}$

FIGURE 12: Simulation results of leaf spring of simulation 2.

\section{Acknowledgments}

The project was supported by the Knowledge Innovation Program of the Chinese Academy of Sciences (Grant no. YYYJ-1122) and the Innovation Program of UAV funded by the Changchun Institute of Optics, Fine Mechanics and Physics, Chinese Academy of Sciences,(CIOMP). The authors also gratefully acknowledge the support from the Science Fund for Young Scholars of the National Natural Science Foundation of China (Grant no. 51305421).

\section{References}

[1] D. Simon, "Hardware-in-the-loop test-bed of an Unmanned Aerial Vehicle using Orccad," in Proceedings of the 6th National Conference on Control Architectures of Robots, 2011.

[2] D. Jung and P. Tsiotras, "Modelling and hardware-in-the-loop simulation for a small unmanned aerial vehicle," in Proceedings of the AIAA Infotech at Aerospace, AIAA Paper 07-2768, Rohnert Park, Calif, USA, May 2007.

[3] P. D. Khapane, "Soimulation of asymmetric landing and typical ground maneuvers for large transport aircraft," Aerospace Science and Technology, vol. 7, no. 8, pp. 611-619, 2003.

[4] K. Ro, "A descriptive modeling and simulation of aircraftrunway dynamics," AIAA Paper 2003-1895, 2003.

[5] A. A. Shabana, Computational Dynamics, John Wieley \& Sons, New York, NY, USA, 2010.
[6] T. Nagata, V. J. Modi, and H. Matsuo, "Dynamics and control of flexible multibody systems part I: general formulation with an order N forward dynamics," Acta Astronautica, vol. 49, no. 11, pp. 581-594, 2001.

[7] K. K. Hauser, C. Shen, and J. F. O’Brien, "Interactive deformation using modal analysis with constraints," in Proceedings of the Graphics Interface Canadian Human-Computer Commnication Society (CIPS '03), pp. 247-255, June 2003.

[8] D. L. James and D. K. Pai, "DyRT: dynamic response textures for real time deformation simulation with graphics hardware," in Proceedings of the 29th Annual Conference on Computer Graphics and Interactive Techniques (SIGGRAPH '02), pp. 582585, July 2002.

[9] F. Naets, T. Tamarozzi, G. H. K. Heirman, and W. Desmet, "Real-time flexible multibody simulation with Global Modal Parameterization," Multibody System Dynamics, vol. 27, no. 3, pp. 267-284, 2012.

[10] W. Krüger, I. Besselink, D. Cowling, D. B. Doan, W. Kortüm, and W. Krabacher, "Aircraft landing gear dynamics: Simulation and control," Vehicle System Dynamics, vol. 28, no. 2-3, pp. 119$158,1997$.

[11] C. Trevor and K. Kevin, "Constrained layer damping test results for aircraft landing gear," Structural Dynamics, vol. 3, pp. 303314, 2011.

[12] M. A. Neto, J. A. C. Ambr'Osio, and R. P. Leal, "Flexible multibody systems models using composite materials components," Multibody System Dynamics, vol. 12, no. 4, pp. 385-405, 2004. 
[13] H. K. Celik, A. E. W. Rennie, and I. Akinci, "Deformation behaviour simulation of an apple under drop case by finite element method," Journal of Food Engineering, vol. 104, no. 2, pp. 293-298, 2011.

[14] D. H. Bae, C. H. Lee, and D. S. Bae, "Non-linear flexible body analysis for mechanical systems," Journal of Mechanical Science and Technology, vol. 26, no. 7, pp. 2159-2162, 2012.

[15] M. R. Hatch, Vibration Simulation Using Matlab and ANSYS, Chapman \& Hall/CRC, 2001.

[16] K. Sherif, W. Witteveen, and K. Mayrhofer, "Quasi-static consideration of high-frequency modes for more efficient flexible multibody simulations," Acta Mechanica, vol. 223, no. 6, pp. 1285-1305, 2012.

[17] E. Bakker, L. Nyborg, and H. B. Pacejka, "Tyre modelling for use in vehicle dynamics studies," Tech. Rep. SAE Paper 870421, 1987.

[18] AGARDograph, Enhancement of Aircraf Ground Handling Simulation Capability, AGARD-AG-333, 1998.

[19] J.-M. Biannic, A. Marcos, M. Jeanneau, and C. Roos, "Nonlinear simplified LFT modelling of an aircraft on ground," in Proceedings of the IEEE International Conference on Control Applications (CCA '06), pp. 2213-2218, Munich, Germany, October 2006.

[20] A. A. Shabana, Dynamics of Multibody Systems, Cambridge University Press, Cambridge, Mass, USA, 3rd edition, 2005. 

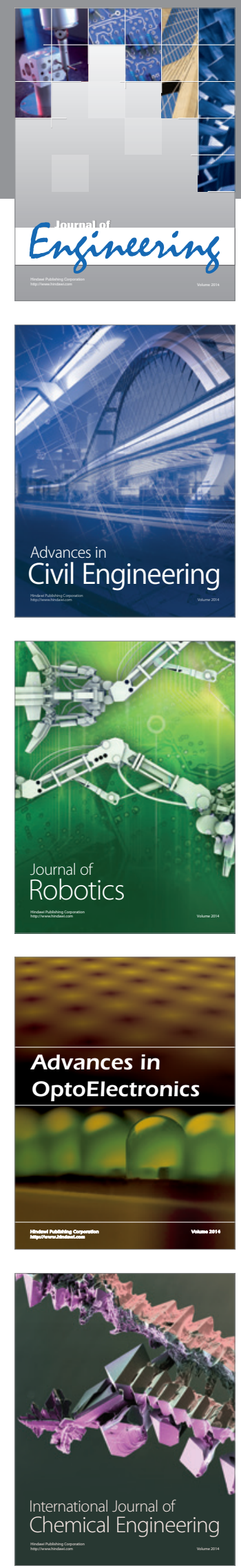

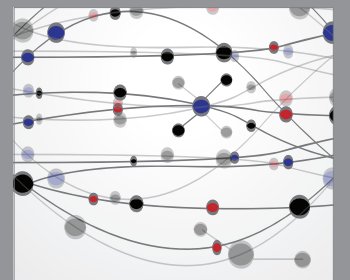

The Scientific World Journal
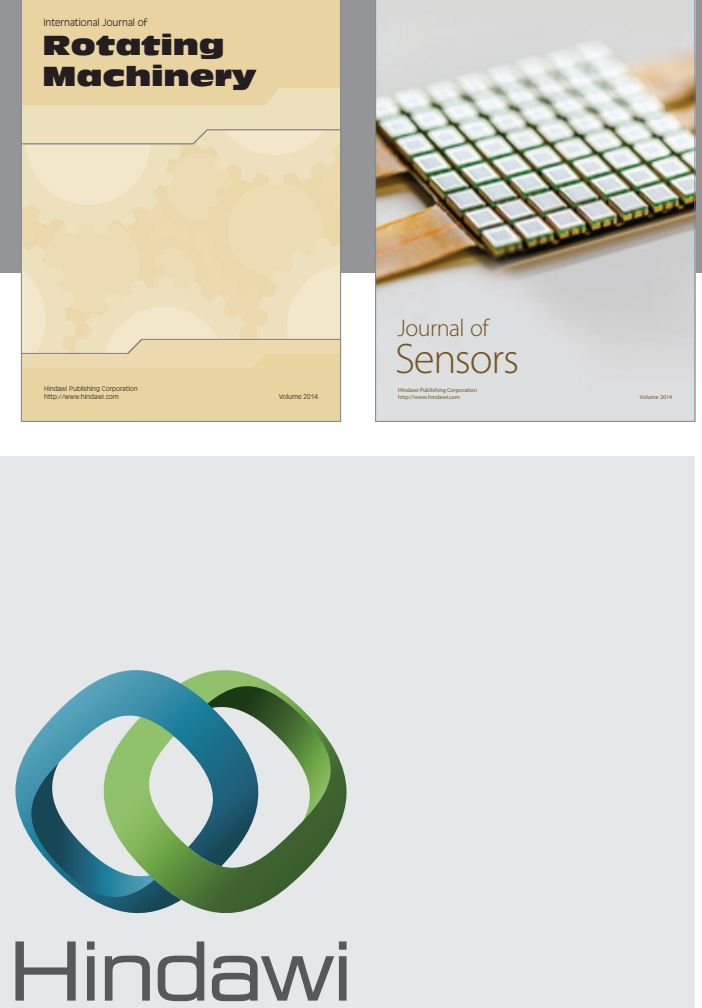

Submit your manuscripts at http://www.hindawi.com
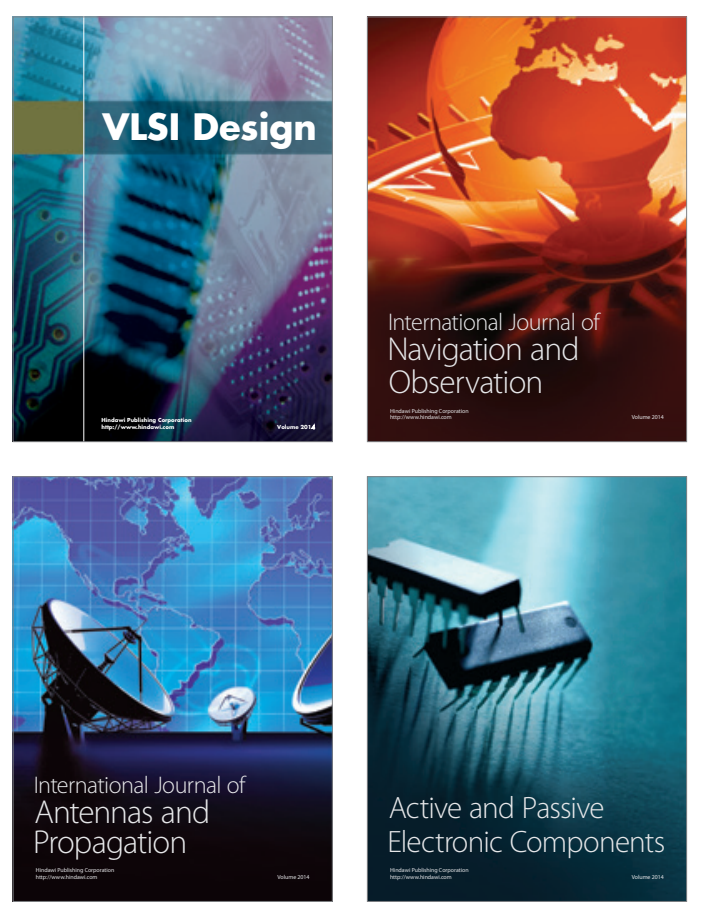
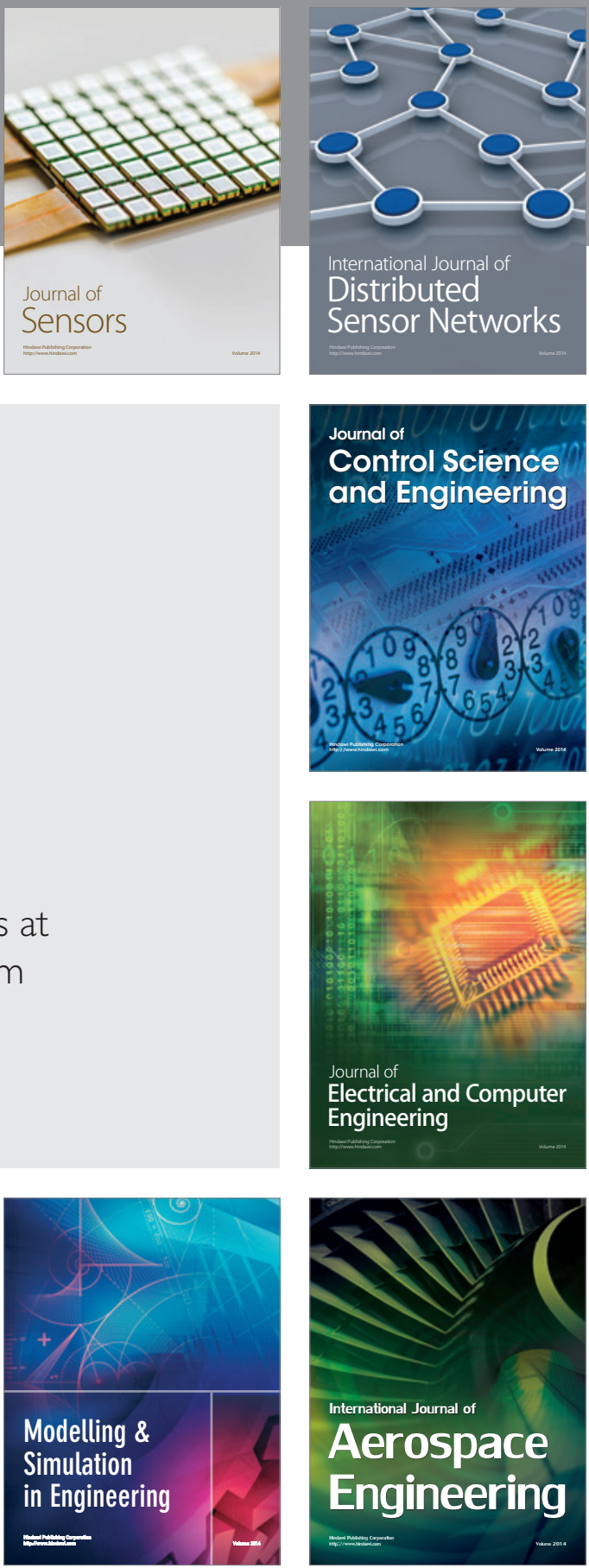

Journal of

Control Science

and Engineering
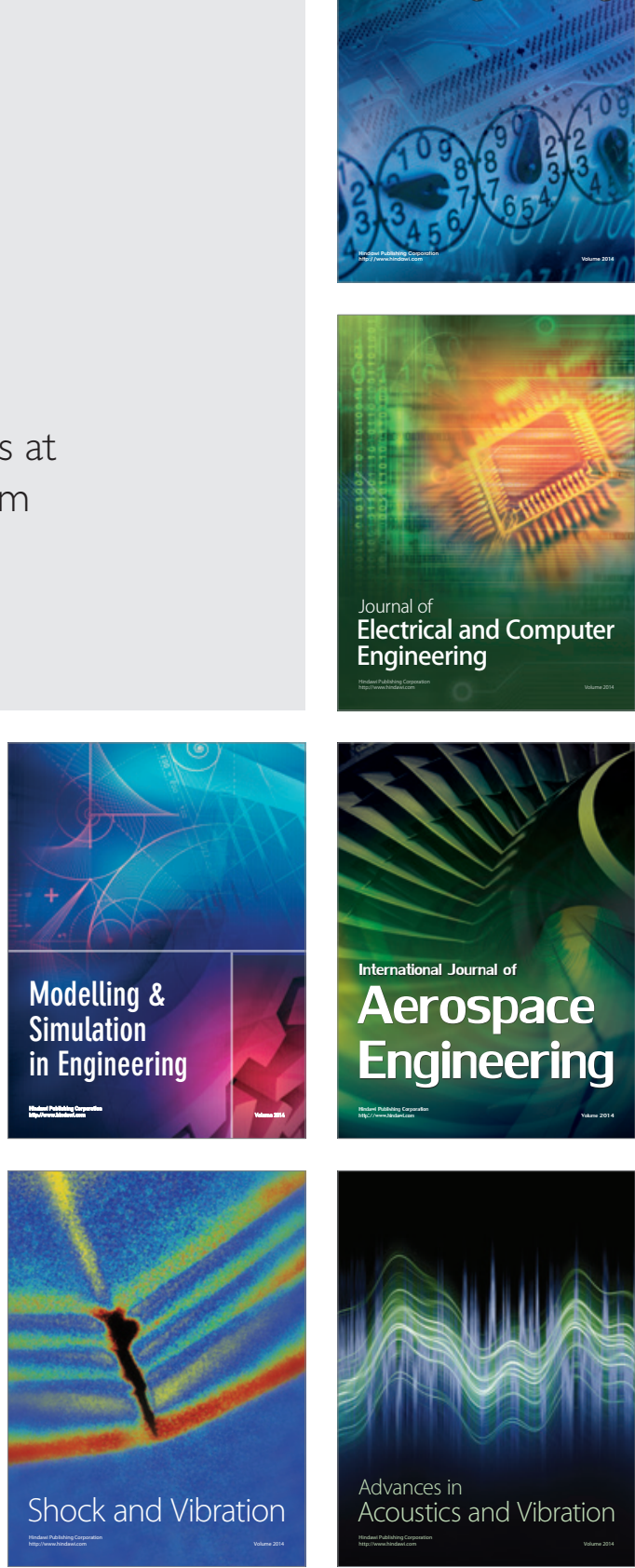